\title{
New routes to kill weeds
}

Nature 559, 415-418 (2018)

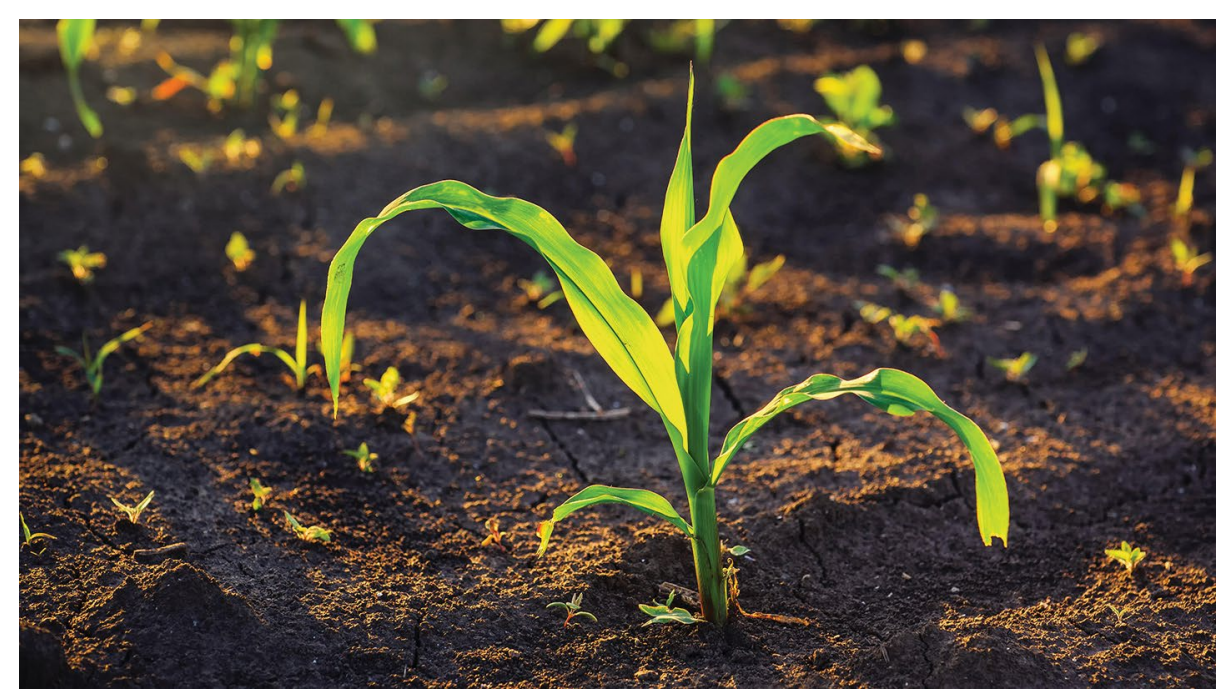

Credit: Igor Stevanovic / Alamy Stock Photo

In agriculture, herbicide application has been widely used to protect crops from weed invasion. However, herbicide treatment could lead to resistance in weeds. Recently, Yi Tang's group and Steven E. Jacobsen's group (both at the University of California, Los Angeles, CA), along with Jiahai Zhou's group (at the Shanghai Institute of Organic Chemistry, CAS, China), collaborated in proposing a new strategy for herbicide discovery and demonstrated a successful attempt in identifying a natural-product herbicide together with its resistance gene.

The branched-chain amino acid (BCAA) biosynthetic pathway performs 'housekeeping' functions in plants and fungi but is non-existent in animals. Yan et al. hypothesized that developing compounds which interrupt the BCAA biosynthetic pathway might be an efficient approach to eliminate plant growth without side effects in animals. Among the enzymes involved in this pathway, dihydroxyacid dehydratase (DHAD) is essential and highly conserved across a broad range of plant species, and thus could serve as a desired target for new herbicides. Yan et al. screened for natural-product biosynthetic gene clusters in multiple fungal genomes, with an assumption that the gene cluster should contain at least one resistant gene homologous to DHAD. Applying this strategy to genomic data mining, they identified a conserved gene cluster in Aspergillus terreus that consists of four genes, ast $A-D$. The ast $D$ gene is the selected homologue of DHAD. Co-expressing ast $A-C$ in yeast yielded a new compound, aspterric acid, which is also a broad-spectrum inhibitor of plant growth at a relative low concentration $(250 \mu \mathrm{M})$. Yan et al. further discovered, through biochemical assays and crystal structure analysis, that aspterric acid specifically targets plant DHADs but not the fungal AstD. Toxicity tests suggest that aspterric acid at a concentration of $500 \mu \mathrm{M}$ has no detectable cytotoxicity in human cell lines. Moreover, they showed that transgenic Arabidopsis plants expressing the fungal ast $D$ gene displayed strong resistance to aspterric acid.

In this work, the researchers introduced a fungal genome screening approach for herbicide discovery and showed its effectiveness by identifying a new herbicide with a desired mode of action as well as the associated resistant gene. In the future, scientists may continue to develop this lead into next-generation herbicides for agricultural application.

\section{Lei Lei}

Published online: 3 August 2018 https://doi.org/10.1038/s41477-018-0227-5 